\title{
Integrated defect sensor for the inspection of fiber-reinforced plastics using air-coupled ultrasound
}

\author{
Yannick Bernhardt and Marc Kreutzbruck \\ Institut für Kunststofftechnik, University of Stuttgart, Stuttgart, 70569, Germany \\ Correspondence: Yannick Bernhardt (yannick.bernhardt@ikt.uni-stuttgart.de)
}

Received: 30 September 2019 - Revised: 5 February 2020 - Accepted: 21 February 2020 - Published: 31 March 2020

\begin{abstract}
Air-coupled ultrasound (ACU) is a non-destructive testing (NDT) method with a rising significance in industrial use. Common cases where ACU is used are the testing of fiber-reinforced plastic or testing of weld joints between metal sheets. The advantage compared to contact ultrasound is the absence of a liquid, solid or gellike couplant. The usage of a couplant is an obstacle for developers of automatic scanning systems for ultrasonic testing because it takes a huge effort to integrate a system that delivers a continuous flow of the couplant. In addition a further step of cleaning is often necessary. ACU needs specially adapted probes to compensate for the tremendous impedance difference between a solid and air. A standard method uses two ACU probes in a normal transmission mode. With slanted probes, it is possible to generate Lamb waves in plate-like materials. Because of the contact to the surrounding air, Lamb waves transmit ultrasound to the air on both sides of the plate continually. These so-called leaky Lamb waves can be used with only one accessible side, and by using a specific resonance angle, a higher signal-to-noise ratio (SNR) is achievable. In the past, the correct angle was determined using an iterative method, where the angle of incidence was changed manually while observing the amplitude level. With the stepper-motor-driven angle scanning system, introduced here, the determination of the resonance angle is possible automatically. The system allows changes of the incidence angle during the ultrasound scan too. This makes it possible to adapt the system to wall thickness changes and changes of the radii of the parts contour.
\end{abstract}

\section{Introduction}

Due to the high demand on environment safety on the modern automotive industry, lightweight design is reaching higher level of importance. By reducing the mass of an airplane structure by about $12 \%$, reductions of fuel consumption of about $7 \%$ are possible (Amendola et al., 2011). Besides the aim to achieve climate goals by $\mathrm{CO}_{2}$ reduction, there is also a cost-driven factor for lightweight design because of the reduction of fuel consumption. Weight reduction can be achieved by the variation of different parameters. One example is to replace materials with a high density with materials with a lower density but with similar performance in stiffness and strength. This can be reached by the usage of fiber-reinforced plastic instead of aluminum alloys in aircraft fuselages. Similarly, lightweight engineers try to achieve lower masses by reducing the material volume. In order to have a correct design, it is necessary to know the attacking loads and the materials properties exactly. By reducing the ratio between bearable loads of the material and the attacking load, lighter designs are possible. Already small defects in the structure can cause catastrophic damage when the safety factors are low. Therefore, the influence of structural damage has to be evaluated very carefully, and periodical testing of structural parts is necessary.

Typical defects in this material class are delaminations, fiber rupture, matrix cracks, inhomogeneities with low fiber content, fiber undulations, pores or trapped particles. In recent years, several non-destructive testing (NDT) methods were developed for testing fiber-reinforced plastics. There are methods only suitable for detection of near-surface defects like shearography (Gregory, 2003) or lock-in thermography (Rahammer, 2018). These methods are usable for most fiber-reinforced lightweight structures due to thin walls. There are also volume methods like X-ray (computed tomography), contact ultrasound (Holmes et al., 2005) or air- 
coupled ultrasound (ACU; Gaal et al., 2012), which is a contact-free testing method.

\section{Air-coupled ultrasound}

For contact-free ultrasound, acoustic waves have to be transmitted through the air directly. This leads to several obstacles that have to be overcome. One main obstacle is the large difference of the acoustic impedance between a solid and air which causes more than $99.9 \%$ signal loss due to reflection. Another main obstacle is that frequencies higher than $1 \mathrm{MHz}$ are not applicable, because the frequency dependent attenuation will lead to signal reduction as well, and the signal will be smaller than the noise.

The problem of the high impedance difference can be resolved by special probe design, high sound pressure and sensitive low-noise amplification (Bhardwaj, 2001). Due to the strong reflections, air-coupled ultrasound is commonly used in normal transmission mode. By using the slanted transmission mode, it is possible to generate Lamb waves and gain several decibels in the signal-to-noise ratio (SNR). The travel speed of Lamb waves depends on excitation frequency, plate thickness and plate stiffness. Lamb waves traveling through the material transfer their energy continuously to the air. This phenomenon is called "leaky Lamb waves". Due to this effect, ultrasonic waves are excited in air on both sides of the specimen. It is possible to place an ultrasonic transducer either on the opposite side of excitation or on the same side (see Fig. 1). If the second transducer is placed at the same side, it is necessary to shield direct reflections from the specimen's surface. Therefore, a beam shield between the two transducers is mandatory. Castaings (Castaings, 1998) has developed a method for using air-coupled ultrasound in single-sided access. In recent years air-coupled ultrasound was developed as an NDT method in industrial use (Hillger, 2014). With a integrated single-sided air-coupled sensor, the incidence angle of the probes can be adjusted automatically, and fast adaptations to new test scenarios are possible.

The angle of incidence $\theta$ (see Fig. 1) for the excitation of a Lamb wave with a specific travel speed in a plate $c_{\text {Lamb }}$ can be calculated using Snell's law (Eq. 1),

$$
\frac{\sin \theta_{1}}{\sin \theta_{2}}=\frac{c_{\mathrm{air}}}{c_{\text {Lamb }}},
$$

where $c_{\text {air }}$ is the velocity of sound in air, $\theta_{1}$ is the incidence angle of the incident wave and $\theta_{2}$ is the angle of the transmitted wave. Lamb waves travel perpendicular to the normal direction. This results in $\sin \left(\theta_{2}\right)=1$ and for the angle of incidence in Eq. (2).

$$
\theta=\sin ^{-1} \frac{c_{\mathrm{air}}}{c_{\mathrm{Lamb}}}
$$

\section{Integrated defect sensor}

\subsection{Beam shield}

The beam shield between both transducers is crucial for good sensor performance. On one side, the reflected and directly transmitted signals need to be shielded (see Fig. 2), to not overdrive the receiving transducer. On the other side, the guided wave in the specimen should not be damped by the high pressure which is applied by the beam shield.

To suppress unwanted sound signals, either reflexion or absorption can be used. By adapting a foam roll to the introduced sensor here, a combination of both shielding mechanisms was used. Inside of the sensors head a 3D-printed reflector shield was assembled (Fig. 3). The foam roll has the advantage to easily roll over the specimen's surface instead of causing too high of a pressure and damping the plate wave. Foam-like beam shields that are sliding over the surface instead of rolling can fold down due to the movement of the sensor head and lose the ability to shield sound waves.

\subsection{Incidence angle control algorithm}

The correct angle of incidence is an important test parameter. Only when the transducers are set to a correct angle are highamplitude dynamic levels possible. Practically a common solution is to manually vary the angle until a high-amplitude level is reached. For an automatic system, the manual way to find the angle which gives the highest amplitude is not a suitable solution. Therefore an automatic angle scanning system was developed. Using two stepper motors, the incidence angle of both transducers is independently adjustable.

There are several ways to find an optimal angle of incidence. The simplest search algorithm is to sweep linearly through the whole angle range and record the amplitude signal in specific small intervals. The angle with the highest amplitude is the optimal angle of incidence. Another possible way is by using a special optimization algorithm to find the signal with the highest amplitude in a faster way. One possible algorithm similar to the bisection method (Munz and Westermann, 2012) is to first define a specific angle interval, in which the highest amplitude is assumed. In Fig. 4 the bisection procedure is shown schematically in a diagram. At first, the amplitude signals on the boundaries and in the center of the interval range are recorded. Second, the amplitude levels of left boundary and right boundary are compared, and the next point is chosen in the middle of the interval between the center and boundary with higher amplitude. With this new points, the method is repeated several times, until the residuum is small enough to assume convergence.

\subsection{Areal scan with air-coupled ultrasound in re-emission}

After setting the correct angle of incidence on a test piece without defects, the next step is to perform a non-destructive 


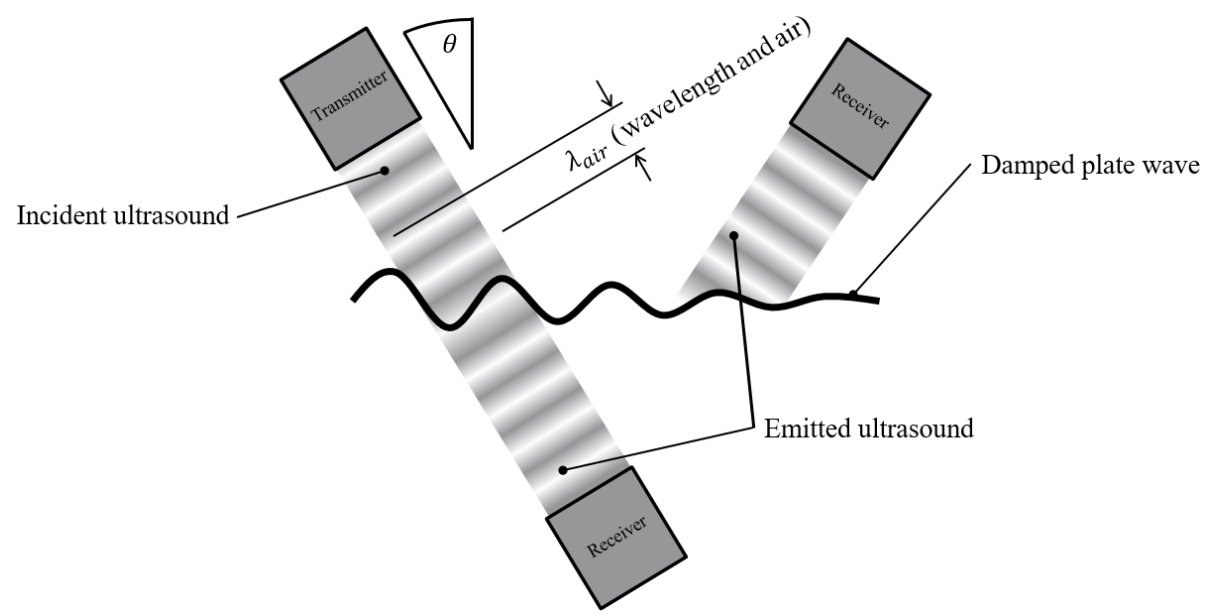

Figure 1. Excitation of Lamb waves with air-coupled ultrasound. The Lamb wave is excited by the transmitter and propagates from the left to right with a velocity of $c_{\text {Lamb. }}$.

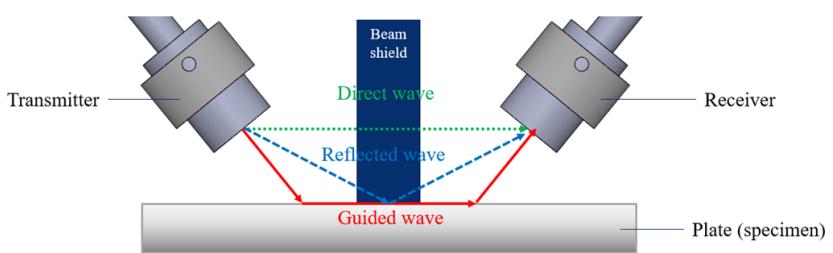

Figure 2. Schematic drawing of a beam shield in re-emission (single-sided) setup. In green, blue and red the different paths of possible wave propagation are shown.

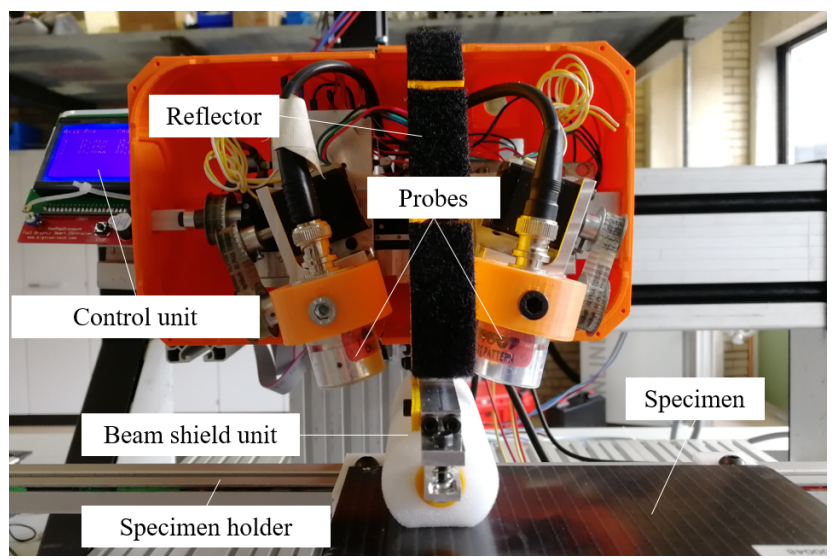

Figure 3. Open ultrasound sensor including beam shield and two slanted ACU probes.

evaluation of the specimen. It is always assumed that the angle optimization was performed on a flawless spot of the specimen. While moving the sensor head over a carbonfiber-reinforced plastic (CFRP) part, the amplitude over time (A scan) changes in relation to the materials stiffness and thickness. Defects usually show different material behavior regarding the stiffness and sometimes thickness. While scan-

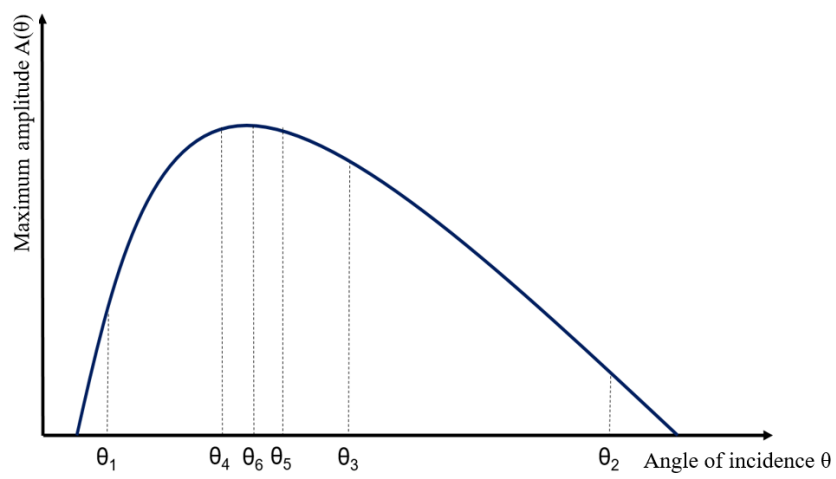

Figure 4. The bisection optimization method for finding the optimal angle of incidence.

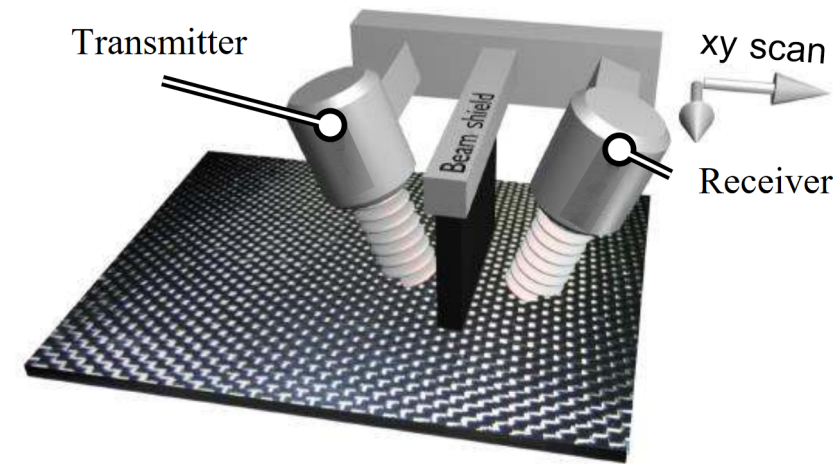

Figure 5. Setup for a C scan in re-emission, where the transmitter and receiver are placed on the same side of the specimen.

ning line by line over a specific area and color-coding the amplitude level in an $x-y$ plot, a defect map is successively created. These color maps are called a $\mathrm{C}$ scan. In Fig. 5 the setup for $\mathrm{C}$ scans in re-emission is shown. 


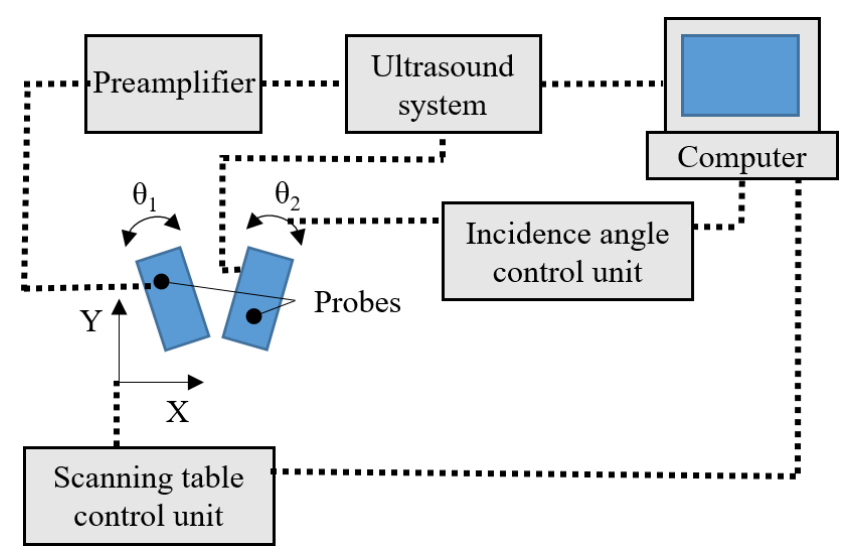

Figure 6. Schematic drawing of the test setup. The angles $\theta_{1}$ and $\theta_{2}$ indicate the angles of incidence between the center axis of the probe and a line normal to the specimen.

\section{Test results and discussion}

The following tests were performed with the air-coupled ultrasound system Airscope TT (Dasel Sistemas, Madrid, Spain) and with two circular, point-focused $200 \mathrm{kHz}$ (focal distance: $38 \mathrm{~mm}$, active diameter: $13 \mathrm{~mm}$ ) NCG200-D13P38 transducers produced by the ultran group (State College, PA, USA). The signal was preamplified using the lownoise amplifier ULTRASONIC PREAMP by Olympus K.K. (Tokyo, Japan). The scans over flat specimens were performed with a three-axis scanning table FLACHBETTEINHEIT by isel ${ }^{\circledR}$ Germany AG (Eichenzell, Germany). The motor control unit for the scanning table is the MotorMOTION system (Dasel Sistemas, Madrid, Spain). The stepper motor controller for the angular scanning system is based on an Arduino Mega2560 controller together with a RAMPS 1.4 stepper motor driver. The controller was programmed so that the motors are controllable either by an external display or via USB. The automatic angle optimization tool was programmed in MATLAB (C) (MathWorks, Natick, MA, USA). The tests were performed with an overall amplification of $85 \mathrm{~dB}$ on the receiver side and with burst excitation on the sender side (pulse train of six rectangular pulses at $200 \mathrm{~V}$ ). A schematic drawing of the whole setup is shown in Fig. 6.

\subsection{Angular scan}

The first tests were performed with a two-parametric-scan method on a flawless $4 \mathrm{~mm}$ thick poly (i.e., polymethyl methacrylate; PMMA) plate. Using the two-parametric scan, the incidence angles were swept independently. Theoretically it is also possible to only change the angles symmetrically. Results in Fig. 7. show that the maximum amplitude is not reached at a symmetrical angle configuration but at $\theta_{1}=10^{\circ}$ and $\theta_{2}=14^{\circ}$ (see Fig. 8). This angle deviation results most probably from differences in the air-coupled ultrasound probes. Switching the positions of the probes showed

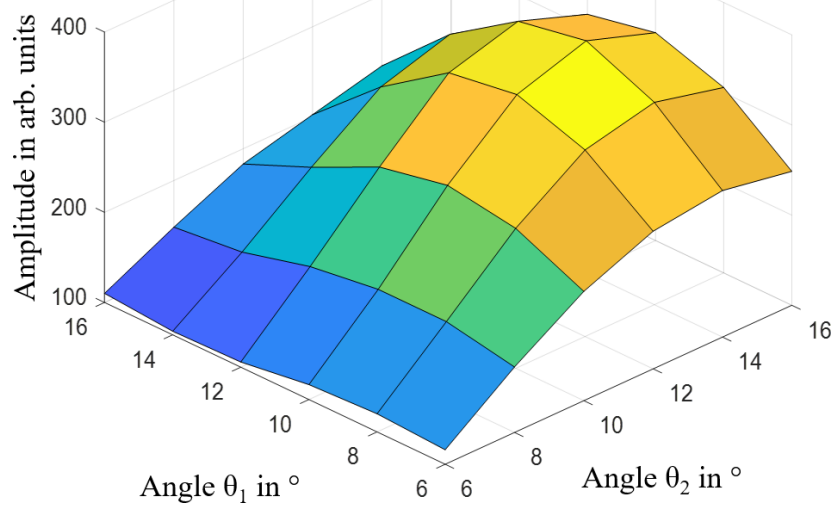

Figure 7. Results of a two-parametric incidence angle scan.

angle deviations in the opposite way. Differences in probe that can influence the optimal angle of incidence can be deviations in frequency because of the frequency dependence of Lamb wave velocity. Another point that can influence the angle of incidence could be differences in the focusing of the probes. Using the symmetrical approach takes less time than the two-parametric scan.

In Fig. 8, the use of the optimization approach via bisection is visualized. The necessary points for finding the optimal angle of incidence are not equally distributed over the angle range. Finding the angle using a symmetrical approach takes less time than a two-parametric scan (10 s compared to $60 \mathrm{~s}$ ), but the angle adjustment has to be done just once, and with the two-parametric scan a higher SNR can be achieved. This can be explained with small differences in the center frequency of the probes. When the setup does not have exactly symmetrical parameters, the optimal angles are not symmetrical.

\subsection{Areal scan}

Quasi-isotropic carbon-fiber-reinforced plastic (CFRP) plates with a thickness of $4 \mathrm{~mm}$ were tested after an impact with an energy of $40 \mathrm{~J}$ (drop test with an impactor with a nose radius of $10 \mathrm{~mm}$ ). A quasi-isotropic layup consists of unidirectional CFRP layers with a fiber orientation at $0,45,-45$ and $90^{\circ}$ with an equal distribution. On the impact side the defect can be barely seen (see Fig. 9a). This kind of damage usually shows large defect areas on the backside. With a single-sided visual inspection, it is not possible to determine the real defect size. In many cases it is even not possible to access the backside. The right image of Fig. 9 shows the results of an aerial ultrasonic scan with air-coupled ultrasound. In comparison to the small impact damage on the photographic image, the dark-blue area in the center of the ultrasonic $\mathrm{C}$ scan shows the real 

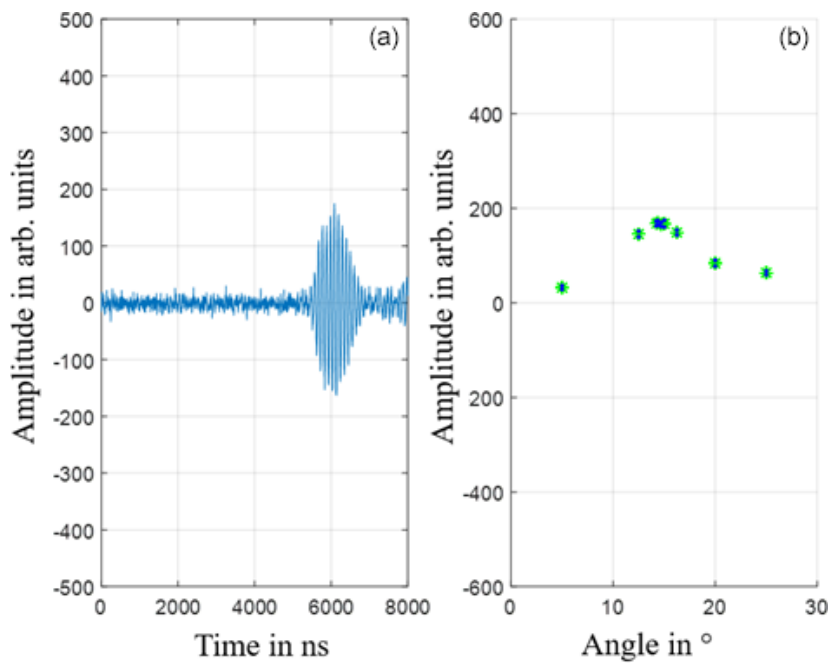

Figure 8. A scan of (amplitude over time) of a Lamb wave (a). Search pattern for the highest amplitude dynamic (b).
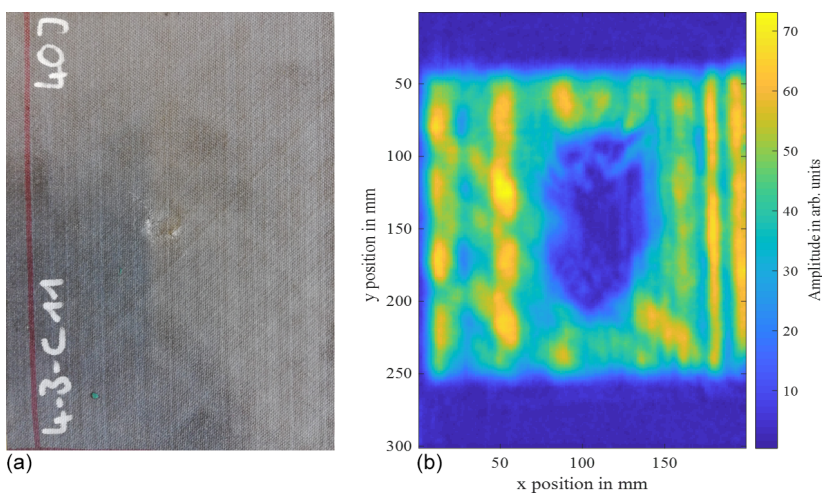

Figure 9. Photography of the tested CFRP plate (a). C scan of the tested plate (b).

defect size. The $\mathrm{C}$ scan is based on the following signal processing: a time window of the first pulse is defined, and the corresponding Fourier transformation is calculated for this time window and its main amplitude. While usually a standard minimum/maximum feature is used, the Fourier approach helps to focus on the amplitude of the main excitation frequency.

The resulting $\mathrm{C}$ scan of the impacted side shows the whole defect area, hidden on the backside. Areas with low amplitude on the top and bottom of the $\mathrm{C}$ scan result from boundary effects due to the small specimen size.

\section{Conclusions}

The sensor head for one-sided ACU testing made it possible to automatically conduct non-destructive testing on CFRP parts. Defects are shown in an amplitude change of the A scan. Even though the ACU probes are not exactly matched, it is still possible to use a symmetrical-angle approach. Better results can be achieved by finding the optimal angle with a two-parametric scan. This procedure takes somewhat longer for finding the optimal amplitude, but the contrast between defect area and non-defect area will be higher. The NDT ACU system is suitable for combination with an automated six-axis robot system and a system for $3 \mathrm{D}$ surface recognition. The latter helps to automatically perform NDT on components, where no CAD information is available or fabrication tolerances are larger than the required positioning of the NDT sensor. Especially when it comes to testing plastic components like CFRP and GFRP (glass-fiberreinforced plastic) in the field of transportation for applications such as aircraft, train, automotive and wind power, the new approach can help to reduce testing time and increase the inspection quality.

Data availability. The data presented in this article are stored in an internal system according to the guidelines of the German Research Foundation (DFG).

Author contributions. YB is responsible for the sample preparation, the construction of the prototype and the measurements. Both authors contributed to the NDT approach and the interpretation of the data. Both authors were involved in the writing process. YB and MK approved the final paper.

Competing interests. The authors declare that they have no conflict of interest.

Special issue statement. This article is part of the special issue "Sensors and Measurement Systems 2019". It is a result of the "Sensoren und Messsysteme 2019, 20. ITG-/GMA-Fachtagung", Nuremberg, Germany, 25-26 June 2019.

Acknowledgements. The underlying research project, ADJUTANT, is funded within the ZIM program of the German Federal Ministry for Economic Affairs and Energy (BMWi) on the basis of a decision by the German Bundestag. The authors would like to express their gratitude for the funding.

Financial support. This research has been supported by the Bundesministerium für Wirtschaft und Energie (grant no. ZF4041123DB7).

This open-access publication was funded by the University of Stuttgart.

Review statement. This paper was edited by Eric Starke and reviewed by two anonymous referees. 


\section{References}

Amendola, A., Iannuzzo, G., Cerreta, P., und Pinto, R.: Future aerostructure for the next generation green civil aircraft, Aerodays 2011, Madrid, 2011.

Bhardwaj, M. C.: Non-Destructive Evaluation: Introduction of NonContact Ultrasound, in: Encyclopedia of Smart Materials, edited by: Schwartz, M., John Wiley \& Sons, New York, 690-714, 2001.

Castaings, M., Cawley, P., Farlow, R., and Hayward, G.: Single Sided Inspection of Composite Materials Using Air Coupled Ultrasound, J. Nondestruct. Eval., 17, 37-45, 1998.

Gaal, M., Döring, J., Bartusch, J., Lange, T., Hillger, W., Brekow, G., und Kreutzbruck, M.: Ferroelektret-Prüfköpfe für berührungslose Ultraschallprüfung von faserverstärkten Kunststoffen, DACH-Jahrestagung 2012 in Graz, 2012.

Gregory, R.: Rapid, Sensitive Inspection of Marine Composites using Laser Shearography, ACMC/SAMPE Conference on Marine Composites Plymouth, 11-12 September 2003, ISBN 1-87091802-09, 2003.
Hillger, W., Bühling, L., and Ilse, D.: Air-coupled Ultrasonic Testing-Method, System and practical Applications, 11th European Conference on Non-Destructive Testing (ECNDT 2014), 6 10 October 2014, Prague, Czech Republic, 2014.

Holmes, C., Drinkwater, B. W., und Wilcox, P. D.: Post-processing of the full matrix of ultrasonic transmit-receive array data for non-destructive evaluation, NDT \& E International, 38, 701-711, ISSN 09638695, 2005.

Munz, C. D. und Westermann, T.: Numerische Behandlung gewöhnlicher und partieller Differenzialgleichungen, SpringerVerlag Berlin Heidelberg, https://doi.org/10.1007/978-3-64224335-6, 2012.

Rahammer, M.: Nachweis von Impact-Schäden in Faserkunststoffverbunden mittels Resonanter Frequenzsweep Thermografie, Dissertation, Universität Stuttgart, Institut für Kunststofftechnik, 2018. 